\title{
A Single-Session Combined Cognitive Bias Modification Training Targeting Attention and Interpretation Biases in Aggression
}

\author{
Nouran AlMoghrabi ${ }^{1,2 *}$ (D), Ingmar H.A. Franken ${ }^{2}$, Birgit Mayer ${ }^{2}$ and Jorg Huijding ${ }^{3}$ \\ ${ }^{1}$ Department of Psychology, Princess Nourah bint Abdulrahman University, Riyadh, Saudi Arabia, ${ }^{2}$ Department of \\ Psychology, Education \& Child Studies, Erasmus University Rotterdam, Rotterdam, the Netherlands and ${ }^{3}$ Department of \\ Child and Adolescent Studies, Utrecht University, Utrecht, the Netherlands \\ ${ }^{*}$ Corresponding author: Nouran AlMoghrabi, Department of Psychology, Princess Nourah bint Abdulrahman University, \\ Airport Road, Riyadh 11671, Saudi Arabia. Email: nhalmoghrabi@pnu.edu.sa
}

(Received 12 December 2019; accepted 5 July 2021; first published online 30 July 2021)

\begin{abstract}
Experimental studies applying cognitive bias modification of attention (CBM-A) and interpretation (CBM-I) to reduce aggression have examined the effect of modifying each cognitive bias in isolation. In order to maximise the potential impact on both biases and symptom reduction, we examined whether a combined bias training procedure targeting both attention and interpretation biases (CBM-AI) in combination would be more effective than targeting interpretation bias (CBM-I) alone. University students (17-35 years) were randomly assigned to either a single session of CBM-AI training $(n=40)$, CBM-I training $(n=40)$, or a control condition $(n=40)$. Contrary to our expectations, participants showed an increase in adaptive attention and pro-social interpretation bias in all training conditions. Additionally, in none of the conditions, we found a significant change on self-reported or behavioural aggression. These findings suggest: (1) that the combined training did not have added effect over single interpretation bias training, (2) that training interpretation bias may lead to changes in attention bias, (3) that elements of the control condition unexpectedly, but interestingly, also affected attention and interpretation biases, and (4) single-session CBM procedures do not produce robust effects on self-report or behavioural measures of aggression in unselected samples.
\end{abstract}

Keywords: attention bias; interpretation bias; cognitive bias modification; aggression

\section{Introduction}

Cognitive models such as the Social Information Processing model (SIP; Crick \& Dodge, 1994) posit that biases in selective attention and interpretation influence the development and maintenance of aggression. Crick and Dodge (1994) hypothesised that aggressive individuals preferentially attend more to hostile than to non-hostile cues in social situations. This attentional bias would increase the likelihood of interpreting social situations in a hostile manner (i.e. interpretation bias), consequently increasing the probability of an aggressive response. Recent studies that used a paradigm known as Cognitive Bias Modification (CBM), that aims at directly influencing a specific cognitive bias, demonstrated that cognitive biases can be trained in the context of aggression (e.g. AlMoghrabi, Huijding, \& Franken, 2018; AlMoghrabi, Huijding, Mayer, \& Franken, 2019; Hawkins \& Cougle, 2013; Hiemstra, de Castro, \& Thomaes, 2018; Penton-Voak et al., 2013; Vassilopoulos, Brouzos, \& Andreou, 2015). In the current study, we developed a new CBM training paradigm in the context of aggression that attempts to maximise efficacy by targeting both attention and interpretation biases (CBM-AI). The goal of the current study was to examine the efficacy of CBM-AI,

(C) The Author(s), 2021. Published by Cambridge University Press on behalf of the Australian Association for Cognitive and Behaviour Therapy. This is an Open Access article, distributed under the terms of the Creative Commons Attribution-NonCommercialNoDerivatives licence (https://creativecommons.org/licenses/by-nc-nd/4.0/), which permits non-commercial re-use, distribution, and reproduction in any medium, provided the original work is unaltered and is properly cited. The written permission of Cambridge University Press must be obtained for commercial re-use or in order to create a derivative work. 
compared with a single-bias CBM training targeting interpretations (CBM-I), and a control condition, in changing attention, interpretation, mood, and aggressive behaviour.

To date, CBM studies have focused primarily on two types of CBM: cognitive bias modification of attention (CBM-A) and cognitive bias modification of interpretation (CBM-I). CBM-A can, for instance, train participant's attention selectivity away from or towards negative or addiction/symptom-related cues (Boffo et al., 2019; MacLeod, Rutherford, Campbell, Ebsworthy, \& Holker, 2002). Most methods that have been used previously to modify and measure attention bias employ a visual probe task to train participants to direct attention away from emotionally negative cues. Each trial presents a negative and a neutral cue followed by a probe (e.g. dot) which replaces one of the cues. Participants have to respond as quickly as possible to the probe. CBM-A using the visual dot-probe task has been used in different research areas such as anxiety (see Bar-Haim, 2010 for review), depression (see Hallion \& Ruscio, 2011 for review), and alcohol and nicotine dependence (see Boffo et al., 2019 for review). A meta-analysis, examining CBM-A's efficacy on both bias and symptom reduction, found that probe-based CBM-A procedures are most promising among anxiety as compared with addiction-related studies (Beard, Sawyer, \& Hofmann, 2012). For instance, CBM-A can result in reductions in attentional bias towards threat cues and reduction in anxiety and stress reactivity (e.g. MacLeod et al., 2002). Beard et al. (2012) suggested that compared with aversive stimuli, it may be more difficult to manipulate attention allocation using appetitive stimuli. Although it seems that probe-based CBM-A procedures are promising in anxiety, the efficacy of this training procedure remains inconsistent (Heeren, Mogoașe, Philippot, \& McNally, 2015). This may have to do with the poor internal reliability of the dot-probe (MacLeod \& Clarke, 2015), and the fact that it only captures inferred effects of attention to visual cues on the screen (Ferrari, Möbius, van Opdorp, Becker, \& Rinck, 2016). This makes it difficult to ascertain whether the training indeed affects key aspects of the visual direction of attention.

Therefore, recent studies explored the possibility of modifying attention bias using an eye-tracker, and found promising results in the context of depression and anxiety with a CBM-A paradigm that trains attention using gaze-contingent techniques (Ferrari et al., 2016; Lazarov, Pine, \& Bar-Haim, 2017; Price, Greven, Siegle, Koster, \& De Raedt, 2016). In this procedure, the screen is updated based on the individual's eye position (Foulsham, Gray, Nasiopoulos, \& Kingstone, 2013) and provides real-time feedback of overt eye movements (Lazarov et al., 2017). This training method ensures attention modification by providing feedback based directly on the gaze direction which aids and improves attention regulation in an adaptive way (Sanchez, Everaert, \& Koster, 2016). This allows to directly target explicit attentional processes associated with different forms of psychopathology (Sanchez-Lopez, De Raedt, van Put, \& Koster, 2019).

This novel attentional training technique seems also very relevant in the context of aggression. For instance, recently it has been found that aggressive individuals take longer time to pay visual attention to relevant social cues (Troop-Gordon, Gordon, Vogel-Ciernia, Ewing Lee, \& Visconti, 2018) and to selectively encode cues (i.e. hostile cues) that fit a hostile interpretation (Horsley, de Castro, \& Van der Schoot, 2010; Troop-Gordon et al., 2018). Thus, the use of the gaze-contingent techniques might help aggressive individuals to effectively attend and encode relevant social cues that help disambiguate the situation. To test this idea, we (AlMoghrabi et al., 2019) developed a novel gaze-contingent CBM-A training aimed at directly targeting attention mechanisms implicated in aggression, by providing feedback contingent on the participant's gaze direction towards adaptive or maladaptive cues. Specifically, the participants received positive feedback if their attention was focused on adaptive cues (i.e. cues that would help disambiguate the situation) for the positive training and maladaptive cues (i.e. negative cues that cannot help disambiguate the situation) for the negative training. Results from the study indicated that compared with the negative training, the positive training successfully increased the fixation duration on adaptive cues over negative cues. These results were in line with previous anxiety and depression gaze-contingent CBM-A training studies that also showed its effectiveness in changing gaze patterns in the intended direction (e.g. Lazarov et al., 2017; Sanchez et al., 2016).

In a similar way to the CBM-A, CBM-I also shows promise. It trains participants to interpret emotionally ambiguous social situations either in a negative or a positive way in order to induce hostile or a 
pro-social bias (Mathews \& Mackintosh, 2000). In anger and aggression studies, CBM-I procedures have mostly used verbal stimuli, such as vignettes or written stories, to train interpretations. These vignettes typically describe hypothetical social situations in which one person harms another, while the intention of the harm-doer is ambiguous. Participants are asked to make responses that require an interpretation of the intention of the harm-doer (whether hostile/intentional or not), after which they receive feedback (correct/incorrect) in order to reinforce/train interpretation in a certain direction. After each assumption, the participant would receive correct/incorrect feedback in order to reinforce the training. This procedure has been shown to successfully induce pro-social interpretations and reduce aggression. For example, Vassilopoulos et al. (2015) trained a sample of 10-12 years old children using flashcards with a short description of ambiguous scenarios. Participants were asked to read a flashcard, and answer a question regarding the situation by choosing one of two sentences that disambiguated the situation in either a pro-social or a hostile way. The benign answer was always reinforced as the correct response. The results showed that three sessions of this interpretation training, effectively increased positive attributions and decreased hostile attributions regarding ambiguous social situations, and decreased self-reported aggression (Vassilopoulos et al., 2015).

Recently, we examined the possibility of modifying hostile attributions using visual stimuli depicting social situations, instead of using written vignettes. Visual non-verbal cues such as facial and physical expressions hold important social information regarding the internal state (including intentions) of the other person. Moreover, it has been suggested that these visual cues could be more meaningful than verbal cues in social interactions (Cadesky, Mota, \& Schachar, 2000). In our study (AlMoghrabi et al., 2018), each image depicted a social situation in which one individual harms another, while the intent of the harm-doer (intentional or unintentional) is ambiguous. Results showed that the positive training led to an increase in pro-social interpretation bias and a decrease in anger and verbal aggression. In addition, the better participants performed on the negative training, the more their interpretations changed in a negative direction and the more aggression they showed on a behavioural aggression task.

As may be clear from the (short and non-exhaustive) overview above, cognitive biases in the context of aggression have exclusively been examined in isolation. However, previous research has demonstrated interrelations between biases in attention and interpretation and has indicated that these biases influence one another in maintaining symptoms (Amir, Bomyea, \& Beard, 2010; Hirsch \& Clark, 2004; Hirsch, Clark, \& Mathews, 2006; White, Suway, Pine, Bar-Haim, \& Fox, 2011). Therefore, a combined CBM training of more than one information processing bias could potentially maximise the symptom reduction as compared with training paradigms that focus on one type of bias at a time (Hirsch et al., 2006). Previous anxiety studies provided evidence for the efficacy of the combined bias training in reducing state and trait anxiety (e.g. Beard, Weisberg, \& Amir, 2011; Brosan, Hoppitt, Shelfer, Sillence, \& Mackintosh, 2011; Lisk, Pile, Haller, Kumari, \& Lau, 2018). For example, Brosan et al. (2011) have provided evidence of the effectiveness of a combined CBM-A and CBM-I to reduce state and trait anxiety in a group of clinically anxious outpatients. Also, Beard et al. (2011) found that a combined CBM-I and CBM-A significantly reduced anxiety symptoms in patients with social anxiety disorder, and the reported effect sizes of the combined CBM trainings were moderate to large. However, those studies have not examined this combination versus single bias training (e.g. Beard et al., 2011; Brosan et al., 2011; Lisk et al., 2018) nor control (e.g. Brosan et al., 2011; Lisk et al., 2018). Therefore, making it more difficult to make strong inferences of the additive efficacy of a combined bias CBM training relative to a single bias CBM training in symptom reduction.

The only study that we are aware of that compared combined bias CBM training to a single bias CBM training and control condition found mixed results (Naim, Kivity, Bar-Haim, \& Huppert, 2018). More specifically, the results indicated that neither the combined CBM-AI, the single bias CBM-A or CBM-I, nor the control condition differed significantly from each other or showed a change in their respective cognitive bias from pre- to post-training. The results showed that only the CBM-A training led to both clinician and self-reported symptom reduction in a sample of social anxiety patients. However, those symptom reductions resulting from the CBM-A training were not associated with a bias change from pre- to post-training. Nonetheless, no previous study to date 
has examined whether this combined training could be of use in the context of aggression. Therefore, it seemed important to investigate whether using the CBM techniques in combination would produce greater effects than using a single bias CBM. In the current study, we compared the efficacy of the combined CBM to a single-bias CBM targeting interpretations, since up until now more studies have provided evidence for the efficacy of CBM-I in aggression reduction (AlMoghrabi et al., 2018; Hawkins \& Cougle, 2013; Penton-Voak et al., 2013; Vassilopoulos et al., 2015).

In the current study, the cognitive bias modification of attention and interpretation (CBM-AI) training provided a first step towards the development of a combined bias training programme aimed at training more adaptive looking and interpreting strategies for aggressive individuals. Previous combined bias training procedures trained participants using two separate tasks such as dot-probe (i.e. to train attention bias) and vignettes (i.e. to train interpretation bias; e.g. Beard et al., 2011; Brosan et al., 2011; Lisk et al., 2018; Naim et al., 2018). In the current study, we trained attention and interpretation bias as part of an integrated training paradigm. In real-life social interactions, aggressive behaviour may emerge not only through hostile interpretation of intent or biases of attention allocation in isolation. These cognitive biases of attention and interpretation could also occur simultaneously in social situations, where both these cognitive biases function as a driving force for aggressive responses. However, the method of vignettes or dot-probe does not allow us to train or examine the role of these cognitive biases in combination when encoding (i.e. attention) social cues and interpreting the intentions of others in social situations. Thus, during the training, participants were presented with pictures of ambiguous social situations in which something unfortunate happens (e.g. one person spilling a drink on someone else). Participants were trained to attend to the facial expression of the harm-doer (i.e. adaptive cue), which can indicate whether the incident happened by accident (or not), and to interpret that situation in a pro-social way.

The aim of the current study was twofold. First, we aimed to examine the effect of a combined CBM-AI training on interpretation and attention bias, and compare this effect with CBM-I training which focuses only on training a single bias (i.e. interpretation bias). Second, we aimed to examine whether there would be additive efficacy of a combined CBM-AI over CBM-I training on aggression reduction.

Regarding the first aim, we expected that training individuals to attend to facial expressions of the harm-doer and to interpret ambiguous situations as pro-social would lead to an increase in adaptive attention and pro-social interpretation bias. Also, we expected that training both attention and interpretation biases would produce more change in a pro-social direction on both targeted biases than training interpretation bias in isolation. Regarding the second aim, we expected that training both attention and interpretation biases would produce a greater aggression reduction than training interpretation bias in isolation. Finally, given that previous findings show that manipulating interpretation (Holmes, Lang, \& Shah, 2009) and attention bias (MacLeod et al., 2002) can also impact mood we included measures of mood before and after the training.

\section{Method}

\section{Participants}

In total, 60 male and 60 female students from Erasmus University Rotterdam (72 Caucasians, 11 Asian, 9 Middle Eastern, 6 Hispanic, 3 African, and 19 others), aged between 17 and 35 ( $M=$ 21.07, $S D=2.50$ ) took part in our experiment in exchange for course credits. Participants were recruited randomly from the university's psychology research website from a list of students who had subscribed to participate in the experiment. All procedures performed in the current study were in accordance with the ethical standards of the Helsinki declaration (World Medical Association, 2001) and with adequate understanding and written consent of the participant.

\section{Stimulus Materials}

Fifty-five different images were used, and each image appeared only once in a trial. For the practice phase, a set of three neutral images (i.e. unrelated to the images used in the CBM training) were 


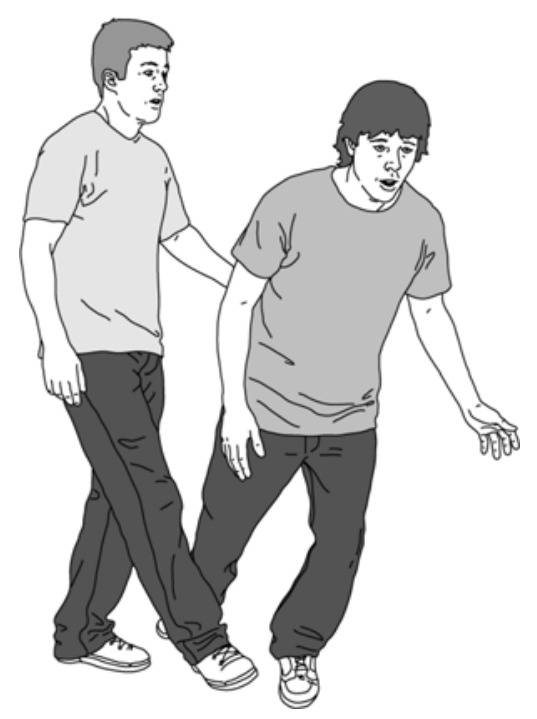

Figure 1. Example image from the assessment phase.

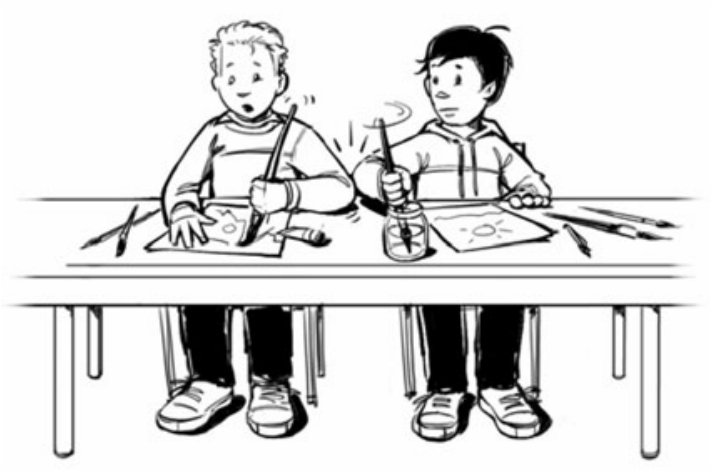

Figure 2. Example image from the training phase.

included. The pre- and post-assessment phases included a set of 12 images from the study of Wilkowski, Robinson, Gordon, and Troop-Gordon (2007; see Figure 1) and were previously used in a similar study by AlMoghrabi et al. (2018). For the training phase, we included a selection of 19 images from the study of Horsley et al. (2010; see Figure 2), AlMoghrabi et al. (2018) and additional 21 pictures collected via stock image websites. Thus, for the assessment and training phases, 52 pictures in total were selected, always containing one adaptive cue and one maladaptive cue. The pictures consist of a variety of hypothetical social interactions that resembles what a normal person would encounter in his daily life. Each image describes interactions between two characters (i.e. male/ male, female/female, or male/female). One of the two characters (i.e. harm-doer) initiates a hostile behaviour (e.g. one person tripping the other) that affects negatively the other character (i.e. victim).

In order to assess the adequacy of the stimulus materials in the training phase, 40 university students were asked to rate the extent to which the depicted harm was intentional and how aggressive is the facial expression of the harm-doer. Participants rated intentionality on a 100-point visual analogue scale (VAS) that was anchored with the labels 'Accidental' on the left and 'Intentional' on the right end. Additionally, participants rated the facial expression of the harm-doer on a 100-point VAS that was anchored with the labels 'Friendly' on the left and 'Aggressive' on the right end. The results of the pilot were as expected, the pictures in the training phase were rated on average as highly 
ambiguous regarding the intent of the harm-doer $[M=50.3, S D=8.5]$, and somewhat less but still fairly ambiguous regarding the facial expression of the harm-doer $[M=42.6, S D=5.1]$. Thus, the intentionality ratings of the pictures varied within and between pictures, suggesting that across all pictures, the facial expression and intention of the character that initiated the harm does not include clear cues whether the act of the harm-doer was on purpose or not.

\section{CBM Training}

The CBM training task consisted of three training conditions: CBM-AI, CBM-I, and control. Each training condition consisted of a total of 55 trials that were presented using E-prime software 2.0 (Psychology Software Tools Inc 2002) for Windows. In all training conditions, participants first completed 3 practice trials, then 6 pre-assessment trials, 40 training trials, 6 post-assessment trials, and finally participants rated how they felt at the moment (happy, angry, sad, and afraid). The practice trials and the pre- and post-assessment phases were identical for all training conditions. For both CBM-I and CBM-AI training conditions, the manipulation of interpretation bias took place during the training phase, while only in CBM-AI also a manipulation of attention bias took place during the training phase. The whole CBM task (i.e. including assessment and training) took approximately 25 min to complete.

\section{Phase 1 (practice)}

In order to familiarise participants with the experimental procedure, participants began each CBM training with three practice trials. During each trial, participants were shown a picture with, for instance, six cars on it, were instructed to fix their gaze on a certain Area of Interest (AOI) on the picture (e.g. the red car) and received performance feedback; 'Correct' if they fixed their gaze on the correct AOI; 'Incorrect' if they fixed their gaze on the incorrect AOI; or 'Too slow' if they did not fix their gaze on any AOI and they had to try again.

\section{Phases 2 (pre-assessment) and 4 (post-assessment)}

In order to examine the effects of the training on interpretation and attention bias, an assessment of interpretation and attention bias was administered pre- and post-training. In total, there were 12 images used for the assessment phases and the participant was presented with one image on each trial. To control for possible order effects, half of the participants within each condition received images 1-6 at pre-assessment and images 7-12 at post-assessment, while for the other half of the participants, the order of the images was reversed.

On each trial, an image was presented preceded with a short description of the situation (e.g. He trips!). To measure attention bias, participants were instructed to look at the image closely (e.g. see Figure 1). Two pre-defined areas of interest (AOI) were embedded within each image. The AOI was defined as a square area $(252 \times 210$ or $336 \times 210$ pixels $)$ that encompasses the negative outcome (maladaptive cue) of the situation (e.g. victim tripping over) and the face of the harm-doer (adaptive cue). During this phase, each image was presented for $5000 \mathrm{~ms}$, while eye movements were recorded automatically using an eye-tracker device. In accordance with previous studies (e.g. AlMoghrabi et al., 2019; Gerdes, Alpers, \& Pauli, 2008; Huijding, Mayer, Koster, \& Muris, 2011), only measurements where participants fixated for at least $80 \mathrm{~ms}$ on any of the pre-defined AOIs (i.e. adaptive and maladaptive cues) were considered as a fixation duration.

In order to measure interpretation bias regarding the intention of the harm-doer (IB-intent), participants were asked to answer the question 'Did this happen by accident or intentionally?' Participants rated the intention of the harm-doer using a 100-point VAS ranging from 'Very likely accidental' $(-50)$ on the left and 'Very likely intentional' $(+50)$ on the right end of the scale. Also, we included an assessment of interpretation bias of facial expressions (IB-facial expressions) of the harm-doer. Participants were asked to answer the question 'How friendly or aggressive is the facial expression of the harm-doer?' Using a 100-point VAS, participants rated the harm-doer's facial expression 
ranging from 'Very friendly' (-50) on the left and 'Very Aggressive' $(+50)$ on the right end of the scale. Participants also rated their feelings in terms of the extent to which they would become angry if such an event happened to them (i.e. perceived anger), using a 100-point VAS ranging from 'Very happy' $(-50)$ on the left and 'Very angry' $(+50)$ on the right end of the scale. During the assessment phases, no corrective feedback was provided.

\section{Phase 3 (training phase)}

For the training phase, participants were randomly assigned to either the CBM-I, CBM-AI or control training, that each consisted of 40 trials. Similar to phases 2 and 4, participants were presented on each trial with an image. In the CBM-I training condition, at first, the images were presented for $2000 \mathrm{~ms}$. Then a short description of the situation was presented below the image. For example, for the image presented in Figure 2, participants read the description 'He ruined his painting'. Following the short description, participants were presented with a pro-social interpretation of the scenario, including a keyword 'It was an ac_ident' and participants were instructed to fill in the missing letter. After the participant completed the sentence with the correct letter, both descriptive and filler sentence remained on screen for another $2000 \mathrm{~ms}$. The next screen displayed a comprehension question, for example, 'Was he determined to ruin his painting?' and participants were asked to select 'Yes' or 'No'. In total, there were 40 comprehension questions. Half of the comprehension questions were phrased in a way that the correct answer would be 'Yes' and for the other half, it would be 'No'. Participants received positive feedback (i.e. Correct) when a pro-social interpretation was endorsed or when a hostile interpretation was rejected, and negative feedback (i.e. Incorrect) when they responded in the opposite direction of the training. The feedback was presented at the top of the screen in bold green (for correct responses) or red font (for incorrect responses) and remained on the screen for $1500 \mathrm{~ms}$, after which the next trial began. This interpretation manipulation procedure was closely tailored after the procedure that was successfully used by Hawkins and Cougle (2013).

The CBM-AI training condition was similar to the CBM-I training condition, but instead of passively viewing the images that appeared at the start of a trial, participants were immediately instructed to fixate on the part of the picture that best indicated whether the incident happened on purpose or by accident (adapted from AlMoghrabi et al. (2018)). This gaze-contingent procedure was used as this would help participants to fixate on the most relevant cues of the social situation more efficiently. When fixating on the adaptive cue (the face of the harm-doer) for $1000 \mathrm{~ms}$ fixations, the word 'Correct' was presented on the screen in bold green font. On the other hand, when fixating on the maladaptive cues (negative outcome) for $1000 \mathrm{~ms}$, the word 'Incorrect' was presented on the screen in bold red font. Feedback words were presented on top of the screen for $2000 \mathrm{~ms}$. Independently of participants' fixation on any of the AOIs, all images were presented for $5000 \mathrm{~ms}$. If participants did not fix their gaze on either AOI within this $5000 \mathrm{~ms}$ timeframe, the text 'Too slow' was presented on the screen in bold blue font, and the participant was allowed to try again. After two failed trials for the participant to fixate on any of the pre-defined AOIs, the trial proceeded to the descriptive and filler sentence and further followed the same procedure as for the CBM-I training condition.

Participants in the control group were presented with the same images as presented in the CBM-I and CBM-AI training conditions. In order to keep the procedure similar to the training condition, the images were presented for $5000 \mathrm{~ms}$, and participants were simply instructed to look closely at the images. Similar to the CBM-I and CBM-AI training conditions, participants then read the same descriptive sentences and were also asked to fill in the missing letter in the sentence and answer the comprehension question. However, the sentences with the missing letter and the comprehension question were different than in the CBM-I and CBM-AI training conditions, as they were unrelated to the intent of the harm-doer or the incident in the image. For example, Figure 2 was presented with the description 'He ruined his painting', accompanied by the filler sentence 'They were p_inting'. Following this, the comprehensive question was presented 'Were they in a painting class?' followed 
by feedback regarding their response. Finally, participants rated how they felt at the moment (happy, angry, sad, and afraid) to assess their internal mood post-training, by dragging an arrow on a 100 -point VAS that was labelled 'Not at all' $(-50)$ and 'Very much' $(+50)$ at the extreme ends of the scale.

\section{Eye-Tracking Assessment}

During all training conditions, eye movements were recorded at pre- and post-assessment phases using an SMI-RED 250 device (Sensomotoric Instruments $\mathrm{GmbH}$, Teltow, Germany). The eye-tracker sampled at a rate of $250 \mathrm{~Hz}$.

The stimuli were presented on a 22 -inch computer screen (screen resolution $1680 \times 1050$ pixels). Participants were seated about $60 \mathrm{~cm}$ away from the screen's centre, and head movements were restricted throughout the CBM training by use of a chin-rest. The size of the image was $1344 \times 777$ pixels. A calibration procedure was performed (9-point calibration and 4-point validation) at the start of the CBM training.

\section{Pre-Measures}

Prior to the CBM training, the present study sought to assess participants on a number of measures of state/trait aggression and mood.

Participants completed a measure of state aggression (20-items in total). Physical aggression (9-items), verbal aggression (4-items), and anger (7-items) were measured using a modified version of the Buss-Perry Aggression Questionnaire (AQ) (Buss \& Perry, 1992). Following the same method used by Farrar and Krcmar (2006), the present study reworded the AQ measure to assess state aggression (cf. AlMoghrabi et al., 2018). Participants were presented with an ambiguous story, which stated: 'Imagine that you just bought something to drink. When you walk outside, somebody bumps into you, spilling your drink over your favourite clothes. As you look at the mess, you hear this person swearing'. Then, participants were instructed to rate the extent of the following $(1=$ totally disagree; $7=$ totally agree). The following items (20-items in total) comprised of items from the AQ that were rephrased. For example, the original AQ item 'I have trouble controlling my temper' was rephrased to 'I would have trouble controlling my temper with this person' to match state aggression. In the current sample, Cronbach's alpha for the AQ total score was 0.89 , physical aggression 0.84 , verbal aggression 0.85 , and anger 0.70 .

Reactive and proactive aggression was measured using the Reactive-Proactive Aggression Questionnaire (RPQ; Raine et al., 2006). Participants responded to items that asked them to rate how often a specific situation happens to them $(0=$ Never, $1=$ Sometimes, and $2=$ Often $)$. There were 11-items indicating reactive aggression (e.g. 'damaged things because you felt mad') and 12 -items indicating proactive aggression (e.g. 'taken things from other students'). In the current sample, Cronbach's alpha was 0.75 for reactive and 0.71 for proactive aggression. Additionally, the participant's state mood was measured pre-training by asking participants to rate how happy, angry, sad, and afraid they felt at the moment. For each emotion, they dragged an arrow on a 100-point VAS ranging from 0 'Not at all' to 100 'Very much'.

\section{Post-Measures}

The participants again completed the reworded trait AQ post-CBM training but with a different ambiguous story, which stated: 'Imagine that you are at the Starbucks working on an assignment. Suddenly, someone bumps into your table, spilling coffee all over your notes. You see that the other person looks really annoyed'. In the current sample, Cronbach's alpha for the AQ total score was 0.91 , physical aggression 0.86 , verbal aggression 0.91 , and anger 0.76 . Additionally, the original hostility subscale of the AQ was added post-training and it includes 8-items in total (e.g. ' $\mathrm{I}$ am 
sometimes eaten up with jealousy'). For each of the items, the participants were instructed to rate the extent $(1=$ extremely uncharacteristic of me; $7=$ extremely characteristic of me). In our sample, Cronbach's alpha was 0.81 .

The Word Sentence Association Paradigm-Hostility (WSAP-H; Dillon, Allan, Cougle, \& Fincham, 2016) is a computerised measure to assess interpretation bias. Participants read 16-items, each item is one sentence long and describes an ambiguous interpersonal social situation (e.g. 'Someone frowns at you'). Each sentence was presented with either a hostile word (e.g. Hostile) or a benign word (e.g. Unaware). Participants are instructed to indicate on a VAS how well the word and the sentence are related from 0 'Not at all related' to 100 'Very related'. Each ambiguous sentence was randomly presented twice, alternatively paired with a benign word (e.g. 'Unhappy') and a hostile-related word (e.g. 'Hostile'). Hostile and benign subscales were formed separately for both the hostile and benign words. In the current sample, Cronbach's alpha was 0.86 for the hostile subscale and 0.88 for the benign subscale.

Trait anger was measured using part B of the Novaco Anger Scale (NAS; Novaco, 1994). This questionnaire comprises 25 -items and assesses the tendency to experience anger over a variety of provocative social situations (e.g. 'Being joked about or teased'). The items are rated on a 5-point scale ranging from 0 (little or no annoyance) to 4 (very angry). In the current sample, Cronbach's alpha was 0.92 . Finally, to measure the affective state of the participants, we used the 20-items Positive Affect and Negative Affect Schedule (PANAS; Watson, Clark, \& Tellegen, 1988). Participants were asked to indicate the extent of specific feelings at the present moment on a 5-point scale ranging from 1 (Slightly) to 5 (Extremely). There were 10-positive emotional states (e.g. interested, inspired), 10-negative states (e.g. upset, guilty), and other 5-items were added to the negative states (e.g. snappy, agitated). In the current sample, Cronbach's alpha for positive affect was 0.87 , and for negative affect 0.86 .

\section{Provocation Induction Task}

The Technical Provocation Paradigm (TPP) was adapted from Panagiotidis et al. (2017) to create a provocative situation that might elicit anger and aggressive behaviour. The task was introduced to the participants as a game where they have to drop balls into a barrel in order to collect virtual golden coins and that the highest collector of these golden coins would win 50 euro as a reward.

Before starting with the game, the experimenter explained to the participants that in order to successfully win the trial they need to direct a ball that moves across the screen into the opening (i.e. a width of $2 \mathrm{~cm}$ ) of a barrel by pulling the joystick. If the participant had three-winning streaks (i.e. dropping the ball successfully into the barrel), they win a virtual golden coin that will be presented at the right corner of the screen. However, if they dropped the ball before the end of the three-winning streaks, the participant losses the streak and they need to restart again but they do not lose the golden coins that they have collected. If the participant dropped the ball into the barrel, they will hear a sound that resembles the sound of a ball dropping successfully, and the participant can see the balls that have been collected on the bottom of the barrel. However, if the participant dropped the ball outside the barrel, a loud crashing sound can be heard and the collected balls in the barrel would disappear.

The TPP task consists of two blocks of 40 trials in each block and was presented using Presentation software (version 20.112.04.17). After every 10 trials, a fixation cross will appear on the screen for $5 \mathrm{~s}$, after which the next trial begins. The whole task (including the two blocks) took 13 min to complete.

The first block was the 'Go' condition in which no technical manipulation was happening and the winning/losing of a trial fully depends on the participants' performance using the joystick. The second block was the 'Fail' block in which the task was manipulated (i.e. 12 out of the 40 trials were manipulated) where the joystick does not respond when the participant pulls it and the ball keeps moving right across the screen without dropping into the barrel. After which a message appears on the screen 'Please move the joystick!'

On each trial, the barrel was positioned in the centre of the screen. Moreover, the ball enters from the left side of the screen and moves in a horizontal line on top of the barrel. During the task, if the 
participant complained about the joystick, the experimenter would act in a surprised manner and would reassure the participant that everything should work perfectly. Finally, participants rated how they felt at the moment (happy, angry, sad, and afraid) to assess their internal mood postprovocation, by dragging an arrow on a 100-point VAS ranging from 0 'Not at all' to 100 'Very much'.

\section{Procedure}

Participants were randomised to either: the CBM-I training condition $(n=40)$, which aimed to increase pro-social interpretation bias of intent; or the CBM-AI condition $(n=40)$, which aimed to increase pro-social interpretation bias of intent and attention bias to pro-social cues; or control condition $(n=40)$, which aimed to not cause any change in interpretation of or attention to the social cues. In all training conditions, the experimenter started with a short introduction and a general explanation of the experiment. Following this, participants started by completing the AQ, RPQ, and mood questionnaires. Then, they received specific instructions regarding the eye-tracking procedure and the CBM training. After this, the participants completed again a mood measure. Then, the experimenter gave instructions regarding the TPP task. Finally, the participants completed again the mood questionnaires, AQ, WSAP-H, NAS, and PANAS questionnaires. The entire experiment took approximately $60 \mathrm{~min}$ to complete.

\section{Data Reduction}

Prior to data analysis, separate mean scores for the ratings of the pre- and post-training interpretation of intent and facial expressions bias were computed. Higher mean scores indicated higher ratings in hostile interpretations of intent and facial expressions of the harm-doer than pro-social interpretations. Similarly, we computed a mean score for the ratings of the pre- and post-training perceived anger score. Higher mean scores on perceived anger indicated higher ratings in feeling angry than in feeling happy. For the WSAP-H measure, we calculated an interpretation bias (WSAP-H (IB)) score by subtracting the mean score of the hostility subscale items from the mean score of the benign subscale items. Higher bias scores of the WSAP-H (IB) measure thus indicated a higher rating of prosocial interpretations than hostile interpretations.

Fixation data recorded by the eye-tracker during the assessment phases were used to calculate the total viewing time of each $\mathrm{AOI}$ as an index of attentional bias. First, we calculated mean total viewing times in ms for the pre-defined AOIs, separately for the adaptive and the maladaptive cues at pre- and post-training. The bias score was then calculated for pre- and post-training attention bias (AB) by subtracting the mean total viewing time at the maladaptive cues from the mean total viewing time at the adaptive cues. Higher AB scores, thus, reflect longer fixations on adaptive (facial) than on maladaptive (negative outcome) cues. Finally, for the TPP task, the participants were asked to pull the joystick towards oneself in order for the ball to drop, as a result the joystick amplitude was continuously recorded in a negative value. In case the participant did not move the joystick, the trial would be recorded as '0'. Following the method used in Panagiotidis et al. (2017), we calculated the peak amplitude of each trial by calculating the maximal deflection of the joystick for each trial. Then, we calculated a separate mean score for the 'Go' and 'Fail' block, by averaging the peak amplitudes for each trial on each block.

\section{Results}

\section{Preliminary Analysis}

To determine the appropriateness of our interpretation bias (IB) and attention bias (AB) measures, we correlated $\mathrm{IB} / \mathrm{AB}$-pre and $\mathrm{IB} / \mathrm{AB}$-post scores with concurrently assessed measures of aggression and hostility (i.e. AQ, NAS, RPQ, WSAP-H, TPP, and VAS state anger). The results indicated that there 
were no significant relations between pre- and post-training attention bias scores with most of the respectively pre- and post-training hostility and aggression-related measures. For pre-training $\mathrm{AB}$ scores, there were only significant correlations with the pre-training AQ subscales verbal aggression and anger. For pre-training IB scores, there was only a significant correlation between IB-intent and pre-training AQ verbal aggression subscale (see Table 1).

\section{Baseline Measures}

Overall, there were no significant differences between participants in the CBM-I, CBM-AI, and control condition in their baseline levels on bias measures (AB, IB-intent, and IB-facial expressions), selfreported aggressive behaviour (AQ and RPQ), and mood. However, compared with the CBM-I condition, participants in the control condition reported a higher level of anger on their mood rating prior to training, $F(2,117)=4.63, p=.012, \eta_{\mathrm{p}}^{2}=.07$. Descriptive statistics for the pre- and post-training measures for each training group are presented in Table 2.

\section{Effects of Training on Interpretation Bias}

To examine the effects of the training on IB-intent, a 2 Assessment (pre- vs. post-treatment) $\times 3$ Group (CBM-I, CBM-AI, and control training) repeated measures ANOVA revealed no significant effect for the crucial interaction, $F(2,117)=0.66, p=0.519, \eta_{\mathrm{p}}^{2}=.01$, or for the main effect of Group, $F(2,117)=0.19, p=0.823, \eta_{\mathrm{p}}^{2}=.00$. However, the main effect of Assessment was significant, $F(1,117)=34.24, p<.001, \eta_{\mathrm{p}}^{2}=.23$ (see Figure 3). For all groups, hostile IB-intent scores decreased significantly from pre- to post-training.

To examine the effects of the training on IB-facial expressions, a 2 Assessment (pre- vs. posttreatment $) \times 3$ Group (CBM-I, CBM-AI, and control training) repeated measures ANOVA was conducted. This analysis revealed no significant interaction for Group-by-Assessment, $F(2,117)=0.13$, $p=0.880, \eta_{\mathrm{p}}^{2}=.00$, as well as no significant main effect for Group, $F(2,117)=0.04, p=0.958, \eta_{\mathrm{p}}^{2}$ $=.00$. However, the analysis revealed a significant main effect of Assessment, $F(1,117)=22.38$, $p<.001, \eta_{\mathrm{p}}^{2}=.16$. Overall, hostile IB-facial expressions decreased significantly from pre- to posttraining (see Figure 4).

To assess the training effects on perceived anger, a 2 Assessment (pre- vs. post-treatment) $\times 3$ Group (CBM-I, CBM-AI, and control training) repeated measures ANOVA was conducted. This analysis revealed no significant interaction for Group-by-Assessment, $F(2,117)=0.40, p=0.668, \eta_{\mathrm{p}}^{2}=.01$, as well as no significant main effect for Group, $F(2,117)=1.07, p=.346, \eta_{p}^{2}=.02$. However, the analysis revealed a significant main effect of Assessment, $F(1,117)=22.76, p<.001, \eta_{p}^{2}=.16$. Overall, perceived anger scores significantly decreased from pre- to post-training.

Additionally, we examined the transfer of effect of the CBM training on the WSAP-H (IB) as an external measure of hostile interpretation bias. One-way ANOVA showed that there was a significant difference between the three training conditions, $F(2,117)=3.10, p=.049, \eta_{\mathrm{p}}^{2}=.05$. The post hoc analysis confirmed that participants in the CBM-AI training condition showed a trend towards significantly higher pro-social interpretation bias in terms of the WSAP-H (IB) than participants in the control condition, $F(2,117)=3.10, p=.058, \eta_{\mathrm{p}}^{2}=.05$. However, in the CBM-I training condition, the scores did not differ significantly from the other training conditions on the WSAP-H (IB) $\left(\mathrm{CBM}-\mathrm{AI}, F(2,117)=3.10, p=0.933, \eta_{\mathrm{p}}^{2}=.05\right.$; Control, $\left.F(2,117)=3.10, p=.128, \eta_{\mathrm{p}}^{2}=.05\right)$.

\section{Effects of Training on Attention Bias}

The 2 Assessment (pre- vs. post-treatment) $\times 3$ Group (CBM-I, CBM-AI, and control training) repeated measures ANOVA revealed no significant Group-by-Assessment interaction, $F(2,117)=$ $1.20, p=.304, \eta_{\mathrm{p}}^{2}=.02$, as well as no significant main effect for Group, $F(2,117)=0.61, p=0.548$, 
Table 1. Correlations between Attention and Interpretation Bias of Intent Scores Pre/Post-Training and Aggression-Related Measures Pre/Post-Training

\begin{tabular}{|c|c|c|}
\hline Measures & Attention bias & Interpretation bias of intent \\
\hline \multicolumn{3}{|l|}{ Pre-training } \\
\hline AQ total score & -.17 & .15 \\
\hline Physical Aggression & -.08 & .13 \\
\hline Verbal Aggression & $-.18^{\star}$ & $.18^{*}$ \\
\hline Anger & $-.21^{\star}$ & .09 \\
\hline Reactive Aggression & -.17 & .09 \\
\hline Proactive Aggression & -.07 & .03 \\
\hline VAS Anger & -.11 & .15 \\
\hline \multicolumn{3}{|l|}{ Post-training } \\
\hline AQ total score & -.12 & .09 \\
\hline Physical Aggression & -.03 & .14 \\
\hline Verbal Aggression & -.17 & .02 \\
\hline Anger & -.13 & .04 \\
\hline Hostility & -.08 & .02 \\
\hline NAS & -.05 & -.05 \\
\hline WSAP-H (IB) & .02 & -.16 \\
\hline TPP-Go & -.06 & -.12 \\
\hline TPP-Fail & -.05 & -.11 \\
\hline VAS Anger & -.02 & .11 \\
\hline
\end{tabular}

Note. $\mathrm{AQ}=$ Aggression Questionnaire; VAS = Visual Analogue Scale; NAS = Novaco Anger Scale; WSAP-H (IB) $=$ Word Sentence Association Paradigm-Hostility (Interpretation Bias); TPP $=$ Technical Provocation Paradigm. ${ }^{*} p<.05$.

$\eta_{\mathrm{p}}^{2}=.01$. However, this analysis revealed a significant main effect of Assessment, $F(1,117)=22.17$, $p<.001, \eta_{\mathrm{p}}^{2}=.16$ (see Figure 5). It was found that, in all training conditions, attention bias became significantly more positive from pre- to post-training, indicated by relatively longer fixation durations on the adaptive cues after training.

We performed an additional analysis to test the relation between IB-intent, IB-facial expressions, perceived anger, and attention bias. First, we calculated separate IB-intent, IB-facial expressions, perceived anger, and attention bias change scores by subtracting the bias score before the training from the bias score after the training. Thus, more positive bias change scores indicate that participant's interpretations of intent and facial expressions and perceived anger became more hostile. In contrast, positive bias change scores for attention bias indicated that participant's attention to adaptive cues increased from pre- to post-training. The correlation analysis showed that the change in IB-intent scores only correlated significantly with IB-facial expressions $(r=0.56, p<.001)$, which suggests that the more pro-social participants' interpretations of intentions became, the more friendly they started to interpret ambiguous facial expressions. However, IB-intent did not correlate significantly with the changes of attention bias $(r=.06, p=0.514)$ and perceived anger $(r=-.05, p=0.556)$. Changes in attentional bias scores were significantly correlated with changes in perceived anger $(r=-.29$, $p=.001)$ : the more participants' attention to pro-social cues increased, the less angry they reported to feel if this negative situation would happen to them. The change in attention bias did not correlate significantly with IB-facial expressions $(r=-.02, p=0.839)$. 
Table 2. Descriptive Statistics for Pre/Post-Training Measures for Each Training Group

\begin{tabular}{|c|c|c|c|c|c|c|}
\hline \multirow[b]{2}{*}{ Measures } & \multicolumn{2}{|c|}{ CBM-I training } & \multicolumn{2}{|c|}{ CBM-Al training } & \multicolumn{2}{|c|}{ Control training } \\
\hline & $M$ & $S D$ & $M$ & $S D$ & $M$ & $S D$ \\
\hline \multicolumn{7}{|l|}{ Pre-training } \\
\hline Perceived anger & 16.25 & 7.70 & 16.99 & 9.07 & 14.49 & 7.95 \\
\hline Aggression Questionnaire & 58.68 & 18.91 & 64.38 & 17.87 & 58.80 & 16.88 \\
\hline Physical Aggression & 23.05 & 10.48 & 25.28 & 8.91 & 21.78 & 8.20 \\
\hline Verbal Aggression & 17.33 & 6.72 & 17.43 & 5.46 & 16.00 & 5.88 \\
\hline Anger & 21.03 & 6.49 & 21.68 & 6.41 & 21.03 & 5.94 \\
\hline Reactive Aggression & 17.23 & 2.95 & 18.43 & 3.19 & 18.73 & 3.40 \\
\hline Proactive Aggression & 13.38 & 2.00 & 13.20 & 1.71 & 13.53 & 1.91 \\
\hline VAS Anger & 2.63 & 4.10 & 7.55 & 15.76 & 10.95 & 13.74 \\
\hline VAS Fear & 7.90 & 14.03 & 12.20 & 18.17 & 9.88 & 13.90 \\
\hline VAS Sadness & 12.38 & 14.79 & 15.55 & 19.43 & 15.53 & 16.66 \\
\hline VAS Happiness & 67.68 & 17.25 & 67.15 & 20.96 & 64.43 & 19.24 \\
\hline \multicolumn{7}{|l|}{ Post-training } \\
\hline Perceived anger & 11.58 & 10.03 & 13.70 & 8.07 & 11.36 & 8.33 \\
\hline VAS Anger & 11.95 & 18.54 & 14.40 & 20.65 & 18.63 & 20.52 \\
\hline VAS Fear & 8.83 & 15.64 & 10.88 & 18.33 & 14.93 & 20.68 \\
\hline VAS Sadness & 17.13 & 21.07 & 23.88 & 25.74 & 17.83 & 20.03 \\
\hline VAS Happiness & 65.10 & 16.32 & 67.43 & 15.35 & 62.35 & 15.94 \\
\hline TPP-Go trial & -134.91 & 53.07 & -152.05 & 43.41 & -149.39 & 39.64 \\
\hline TPP-Fail trial & -108.62 & 39.57 & -114.83 & 25.85 & -110.83 & 32.28 \\
\hline VAS Anger post-TPP & 14.83 & 17.11 & 16.95 & 19.90 & 18.68 & 18.82 \\
\hline VAS Fear post-TPP & 3.25 & 8.21 & 6.65 & 12.21 & 3.95 & 7.51 \\
\hline VAS Sadness post-TPP & 10.10 & 18.89 & 16.58 & 19.31 & 9.35 & 10.42 \\
\hline VAS Happiness post-TPP & 64.18 & 18.51 & 65.08 & 21.07 & 60.5 & 19.95 \\
\hline Aggression Questionnaire & 61.40 & 19.93 & 64.75 & 20.41 & 59.98 & 20.61 \\
\hline Physical Aggression & 23.05 & 10.48 & 24.68 & 9.26 & 22.00 & 10.37 \\
\hline Verbal Aggression & 17.33 & 6.72 & 17.40 & 6.36 & 16.15 & 6.61 \\
\hline Anger & 21.03 & 6.49 & 22.68 & 7.24 & 21.83 & 7.08 \\
\hline Hostility & 23.65 & 8.28 & 25.50 & 9.48 & 25.30 & 9.29 \\
\hline NAS & 69.85 & 14.12 & 71.50 & 17.27 & 71.48 & 15.15 \\
\hline PANAS-positive & 26.10 & 8.06 & 26.88 & 6.64 & 26.00 & 7.00 \\
\hline PANAS-negative & 19.53 & 4.72 & 21.93 & 6.60 & 21.38 & 6.67 \\
\hline
\end{tabular}

Note. VAS = Visual Analogue Scale; TPP = Technical Provocation Paradigm; PANAS = Positive Affect and Negative Affect Schedule; NAS = Novaco Anger Scale.

\section{Effects of Training on Mood}

To investigate whether the training affected participants' mood, ratings of the VAS state mood (happy, angry, sad, and afraid) were subjected to a 3 Assessment (pre-CBM training vs. post-CBM training vs. 


\section{IB-hostile intent}

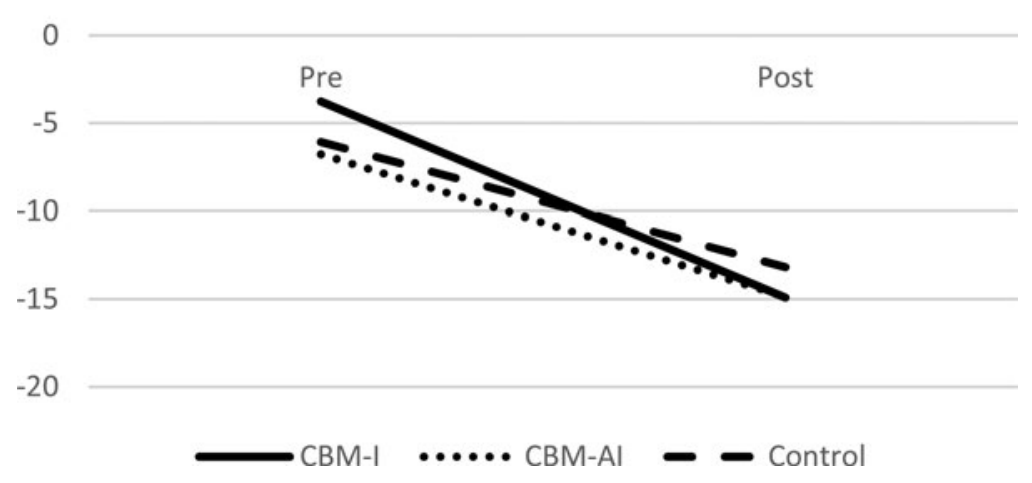

Figure 3. Average interpretation bias of intent scores at pre- and post-training for each training condition.

\section{IB-facial expressions}

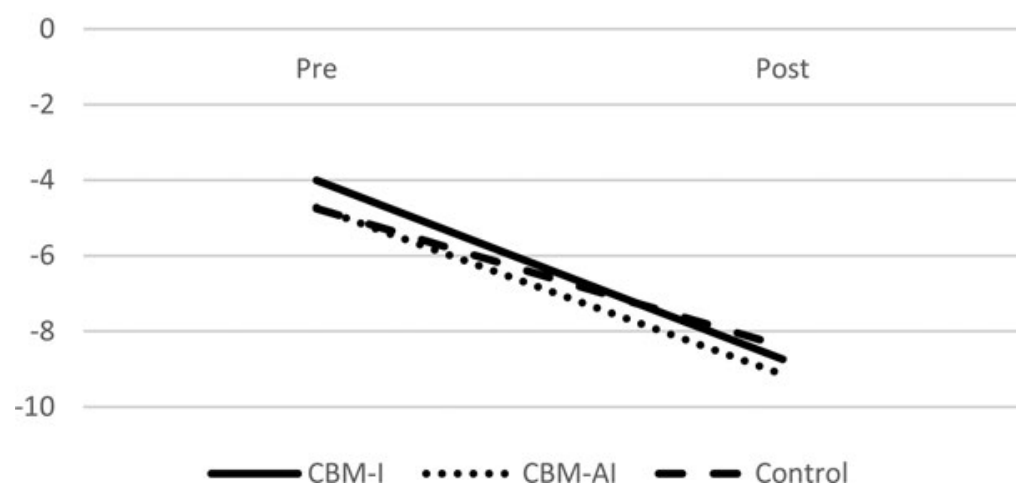

Figure 4. Average interpretation bias of facial expressions scores at pre- and post-training for each training condition.

post-TPP task) $\times 3$ Group (CBM-I, CBM-AI, and control training) repeated measures ANOVA. Overall, the analysis revealed no significant interaction for Group-by-Assessment, all $F<1.38$, all $p>.247, \eta_{\mathrm{p}}^{2}>.00$, as well as no significant main effect of Group, all $F(2,117)<2.74, p>.069, \eta_{\mathrm{p}}^{2}>.01$. Only a significant main effect of Assessment emerged for self-reported anger, $F(2,234)=13.99$, $p<.001, \eta_{\mathrm{p}}^{2}=.11$, self-reported sadness, $F(2,234)=10.73, p<.001, \eta_{\mathrm{p}}^{2}=.08$, and self-reported fear, $F(1.71,199.45)=13.87, p<.001, \eta_{\mathrm{p}}^{2}=.11$. This indicates that, in all training conditions, self-reported sadness and fear decreased from pre- to post-training, while self-reported anger increased from pre- to post-training (see Table 2 for mean scores).

In addition, differences between groups in positive and negative trait affect scores post-training were examined using one-way ANOVA. There were no significant differences between groups in either positive, $F(2,117)=1.172, p=.183, \eta_{\mathrm{p}}^{2}=.00$, or negative, $F(2,117)=.174, p=0.840, \eta_{\mathrm{p}}^{2}=.03$ trait affect scores.

\section{Effects of Training on Aggression}

To investigate training effects on aggression, participants' scores from the AQ were subjected to a 2 Assessment (pre- vs. post-treatment) $\times 3$ Group (CBM-I, CBM-AI, and control training) repeated 


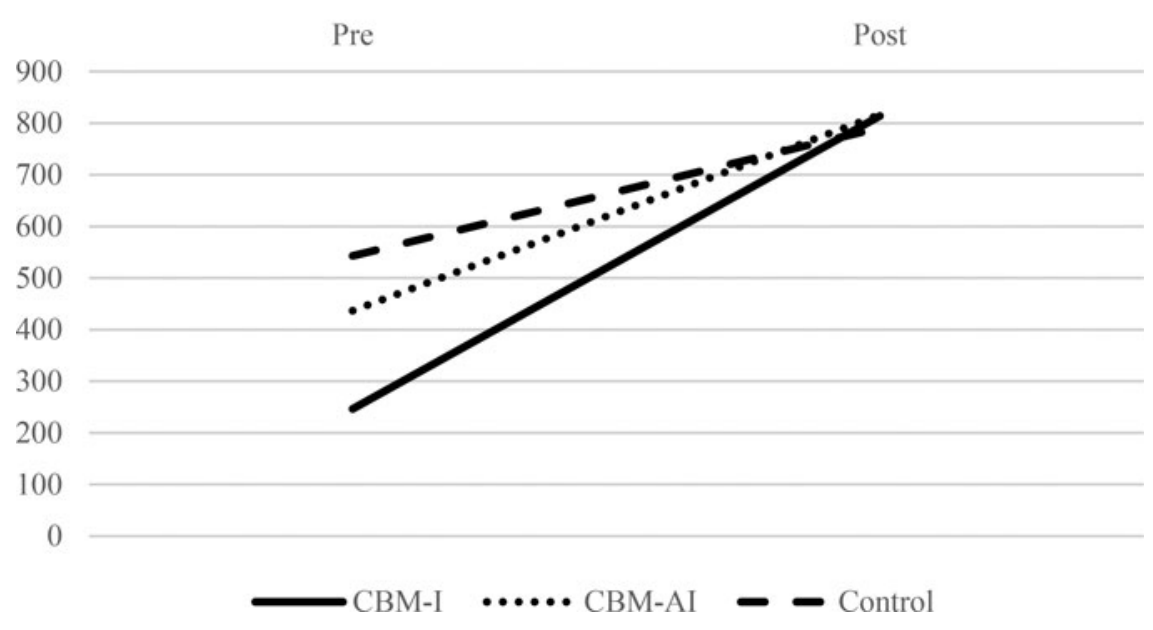

Figure 5. Average attentional bias scores at pre- and post-training for each training condition.

measures ANOVA. The analysis of the AQ total score and its subscales revealed no significant interaction for Group-by-Assessment, for all $F(2,117)<2.20, p>.116, \eta_{\mathrm{p}}^{2}>.01$, as well as no significant main effects of Assessment and Group, for all: Assessment, $F(1,117)<2.92, p>.090, \eta_{\mathrm{p}}^{2}>.02$; Group, $F(2,117)<1.27, p>.284, \eta_{\mathrm{p}}^{2}>.01$.

Next, differences between groups on the AQ hostility subscale and NAS anger scores post-training were examined using one-way ANOVA. The results showed that all training conditions did not differ significantly in terms of their hostility subscale, $F(2,117)=0.51, p=0.605, \eta_{\mathrm{p}}^{2}=.01$, or NAS scoring, $F(2,117)=0.15, p=0.863, \eta_{\mathrm{p}}^{2}=.00$. Additionally, participant's scores on the provocation task (i.e. TPP) were compared between the three training conditions. The results showed that all training groups did not differ in their performance on the provocation task on both trials: trial Go, $F(2,117)=1.63, p=.201, \eta_{\mathrm{p}}^{2}=.03$, and trial Fail, $F(2,117)=.36, p=0.696, \eta_{\mathrm{p}}^{2}=.01$.

\section{Post Hoc Statistical Power Analysis}

To check whether our non-significant results could be due to the lack of statistical power, we conducted post hoc power analysis using $G^{*}$ Power 3.1 (Faul, Erdfelder, Lang, \& Buchner, 2007) for our main effect of interest: the interaction of a $3 \times 2$ RM-ANOVA, with a sample size of 120 , a power of 0.80 , and alpha set to .05 . The power analysis showed that we should be able to detect effect sizes between .14-.18 and larger (i.e. a small to medium effect, according to Cohen's (1969) effect size conventions). Thus, our sample of 120 participants in the current study seems to have been adequate to find practically meaningful effects.

\section{Discussion}

The current study examined whether a single session of a combined cognitive bias modification $(\mathrm{CBM})$ targeting both attention and interpretation biases (CBM-AI) influences attention, interpretation of intent, mood, and aggressive behaviour in a student sample. Additionally, the current study compared the efficacy of the combined bias training to a single-bias CBM training targeting interpretations (CBM-I). The results indicated that a single session of CBM-AI training designed to increase adaptive attention and pro-social interpretation bias indeed increased attention allocation to adaptive cues and pro-social interpretation bias of both intent and facial expressions. However, 
contrary to our expectations, we found that those changes are not significantly different from the bias change in the CBM-I and control conditions. Additionally, in all training conditions, an increased selfreported state anger was found.

\section{Effect on Interpretation and Attention Biases}

Our results showed that the CBM-AI training led to a significant increase in both attentional allocation to adaptive cues and an increase in pro-social interpretation bias of intent. These results are consistent with prior anxiety research, suggesting that CBM-A and CBM-I can be used jointly to modify both attention and interpretation biases (e.g. Brosan et al., 2011). However, we found little evidence that the combined CBM-AI training had a stronger impact on either attention bias or interpretation bias than the CBM-I training or even the control condition. Our results are similar to a previous study that also did not find a greater change in bias after a combined CBM-A and CBM-I training compared with a single bias CBM training or control condition in the context of social anxiety (e.g. Naim et al., 2018). Perhaps the most surprising was the finding that the changes in bias in the control condition were comparable to those in the training conditions. Although this could indicate that the effects in all conditions were simply due to test-retest effects, the fact that we found clear effects in previous studies using similar procedures (AlMoghrabi et al., 2018, 2019) makes this seem rather unlikely. Instead, the design of the control condition may unintendedly cause a change in both attention and interpretation biases. That is, in the control condition, an image was first presented, and the participant was instructed to look closely at the image. Then, after $5 \mathrm{~s}$, the descriptive and filler sentences were presented on the screen below the image. This gave participants ample time to take in all aspects of the scene and prevent them from quickly interpreting its meaning in a hostile way. Thus, the control condition may inadvertently have been a 'training' of sorts as well. This highlights the importance of taking into account the possible influences of specific procedural details (e.g. instructions provided in the training, presentation time of the stimulus material, or feedback) on the efficacy of the CBM training paradigms. More research is needed on the key procedural details influencing the CBM training outcomes in aggression. Also, future work should include a different, truly neutral, control condition before drawing any firm conclusions related to the efficacy of the combined bias training.

The finding that the CBM-I and CBM-AI training conditions led to similar increases in attention allocation to adaptive cues is in line with previous findings in the context of anxiety, and supports the idea that cognitive biases are interrelated and that training one cognitive bias can have an effect on the other (e.g. Amir et al., 2010; Hirsch \& Clark, 2004; Hirsch et al., 2006; White et al., 2011). Interestingly, in the context of aggression, the transfer effects between trained cognitive biases in interpretation and attention do not seem to be bidirectional. That is, in a previous study, changes in attention allocation following a CBM-A training paradigm did not generalise to changes in interpretation bias in aggression (e.g. AlMoghrabi et al., 2019). Research in this area is still scarce and further research is still needed.

The current study extended the findings of AlMoghrabi et al. (2018) by examining the effect of combined bias training on interpretation bias of facial expressions. Previously, it has been shown that aggressive individuals tend to interpret ambiguous facial expressions more negatively than other people (e.g. Schönenberg \& Jusyte, 2014). Overall, the results of the current study show that pro-social interpretation bias of facial expressions increased from pre- to post-training for all training conditions. Moreover, we found an association between changes in both interpretation of intent and interpretation bias of facial expressions. These results are in contrast with the findings of Hiemstra et al. (2018). While they successfully modified hostile interpretations of facial expressions in an aggressive sample using morphed faces, those changes did not generalise to changes in participants' interpretation of intent in a game context. The authors concluded that CBM is more effective in modifying the targeted bias but that those modifications fail to affect other forms of biases. Our results suggest that the effects of CBM on interpretation of intent can generalise to another type of interpretation like the interpretation of facial expressions. It would be interesting for future studies to further examine the 
underlying mechanisms of interpretation of facial expressions, as this knowledge may increase the potential effects of future CBM-I intervention programmes.

\section{Effect on Aggression and Mood}

Contrary to our expectations, the combined CBM training was not more effective than a single bias training and control condition in symptom reduction. In general, none of the training conditions had any effect on the aggression measures post-training. Based on previous single bias training studies targeting interpretation bias (i.e. CBM-I) that showed that this training method can be successful in decreasing anger and aggression (Hawkins \& Cougle, 2013; Vassilopoulos et al., 2015), we expected that changes on both biases of attention and interpretation in a single training procedure would have a stronger effect on aggression. On the contrary, our results are more in line with Naim et al. (2018) who found that a combined bias training, compared with a single bias training and a control condition, did not lead to clinician-reported or self-reported symptom reduction in a sample of social anxiety patients. However, compared with the results of the current study where participants showed bias change, in the study of Naim et al. (2018), none of the training groups showed bias change from pre- to post-training.

Our results further showed that participants in all training conditions reported a reduction in perceived anger in response to a potential provocation situation. This result is in line with a previous study, where high aggressive children reported a decrease in perceived anger in response to hypothetical provocative social situations after taking part in a multi-session attribution modification training (Vassilopoulos et al., 2015). Interestingly, in the current study, the decrease in perceived anger was related to the change in attention bias rather than interpretation bias. That is, the more participants looked at adaptive cues the less they perceived anger in the social situation. This finding is consistent with previous studies that linked trait anger to attention bias to negative cues (e.g. Wilkowski \& Robinson, 2008). However, the reduction in perceived anger did not generalise to anger mood post the provocation behavioural task, since self-reported anger increased in all training conditions.

A possible explanation for the limited effects of our training on aggression and self-reported anger is that, although pro-social interpretation bias of intent increased in all training conditions, the changes in interpretation bias of intent were too modest to have an effect on our measures of aggression. A second possibility is that the increase in self-reported anger was caused by certain features of the training task (e.g. length of the training, negative reinforcement for incorrect responses, cf. Hawkins \& Cougle, 2013). In line with this, all training groups increased in their self-reported anger from pre- to post-training. A related limitation might be that the training conditions differed in baseline anger. Nevertheless, the general increase in anger may have washed out any positive effects of the CBM training on self-reported and behavioural aggression measures. Another possibility is that the currently used behavioural measure of aggression (i.e. TPP) provokes aggression by other processes than those targeted during the training. Most importantly, the TPP provokes aggression in a non-social context, while our training was focused specifically on changing the processing of social information. As a result, the TPP may not be sensitive to the effect of our current training on interpersonal aggression. In line with this, in the study of Hawkins and Cougle (2013), in which they did find an effect of CBM-I on a behavioural measure of aggression, aggression was assessed using an interpersonal task (i.e. response to an insult). This highlights the importance of selecting a (behavioural) aggression measure that taps the trained processes, and that the effects of training do not simply generalise to aggression in any context.

\section{Limitations}

The current results should be taken in light of several limitations. First, the current experiment was conducted in an unselected student sample. Therefore, the current results cannot generalise to an aggressive sample. Somewhat related to this, our sample may have been limited by a restricted range of aggression scores, since we recruited an unselected sample rather than choosing participants based on high or low aggression scores. This could have limited the ability to detect any effect of the 
CBM training on the aggression measures post-training. Thus, the lower levels of aggression could possibly account for the lack of correlation between aggression, attention, and interpretation bias measures. Further research with a clinical or high aggressive sample is necessary to further explore the efficacy of CBM training paradigms on aggression. Second, participants completed a single session of the CBM training. Although all training conditions showed a cognitive bias change in interpretation and attention bias from pre- to post-training, the single training set-up may have been insufficient to differentially affect aggression-related measures. Previous combined bias training studies trained participants over 4 sessions (Brosan et al., 2011), 8 sessions (Beard et al., 2011), and up to 12 sessions (Lisk et al., 2018) and found great symptom reduction. However, those results are inconsistent since Naim et al. (2018) included 8 sessions of combined training and the results indicated that there were no symptom reductions for anxious participants. Future studies should examine the effect of the number of sessions that are necessary to achieve the strongest symptom change.

Third, although we used a similar, but distinct, interpretation bias measure (WSAP-H), we did not include a distinct measure for attention bias. It would clearly be of interest to see if the bias change in the combined training would have transferred not only for interpretation bias but also for attention bias to a different task.

Finally, in the current study, we compared the combined bias training to a single bias training targeting interpretation bias, but not to a single bias training targeting attention bias. Somewhat related to this, the current set-up did not allow us to disentangle what part of the combined training was more effective. In a number of studies, it has been reported that participants experienced CBM-I training more helpful than CBM-A training (e.g. Beard et al., 2011; Brosan et al., 2011; Lisk et al., 2018). This knowledge is important, as knowing the precise way of combining both biases in a training could increase the effectiveness of both training tasks which might maximise the effects of the combined training. Future studies that compare the combined bias training to a single bias training targeting attention and interpretation could provide answers to this question.

\section{Conclusion}

This is one of the first studies that developed and tested a combined bias training procedure targeting both attention and interpretation biases and compared it to a single-bias training targeting interpretations, in the context of aggression. We did not find evidence that the combined bias training is more effective than a single bias training or control condition. We did find suggestive evidence that interpretation training might have a broader cognitive effect on biases of attention and facial expressions.

Declaration of Interest. None.

\section{References}

AlMoghrabi N, Huijding J and Franken IH (2018). The effects of a novel hostile interpretation bias modification paradigm on hostile interpretations, mood, and aggressive behavior. Journal of Behavior Therapy and Experimental Psychiatry, 58, 36-42.

AlMoghrabi N, Huijding J, Mayer B and Franken IH (2019). Gaze-contingent attention bias modification training and its effect on attention, interpretations, mood, and aggressive behavior. Cognitive Therapy and Research, 43, 861-873.

Amir N, Bomyea J and Beard C (2010). The effect of single-session interpretation modification on attention bias in socially anxious individuals. Journal of Anxiety Disorders, 24, 178-182.

Bar-Haim Y (2010). Research review: Attention bias modification (ABM): A novel treatment for anxiety disorders. Journal of Child Psychology and Psychiatry, 51, 859-870.

Beard C, Weisberg RB and Amir N (2011). Combined cognitive bias modification treatment for social anxiety disorder: A pilot trial. Depression and Anxiety, 28, 981-988.

Beard C, Sawyer AT and Hofmann SG (2012). Efficacy of attention bias modification using threat and appetitive stimuli: A meta-analytic review. Behavior Therapy, 43, 724-740.

Boffo M, Zerhouni O, Gronau QF, van Beek RJ, Nikolaou K, Marsman M and Wiers RW (2019). Cognitive bias modification for behavior change in alcohol and smoking addiction: Bayesian meta-analysis of individual participant data. Neuropsychology Review, 29, 52-78. 
Brosan L, Hoppitt L, Shelfer L, Sillence A and Mackintosh B (2011). Cognitive bias modification for attention and interpretation reduces trait and state anxiety in anxious patients referred to an out-patient service: Results from a pilot study. Journal of Behavior Therapy and Experimental Psychiatry, 42, 258-264.

Buss AH and Perry M (1992). The aggression questionnaire. Journal of Personality and Social Psychology, 63, 452-459.

Cadesky EB, Mota VL and Schachar RJ (2000). Beyond words: How do children with ADHD and/or conduct problems process nonverbal information about affect? Journal of the American Academy of Child \& Adolescent Psychiatry, 39, $1160-1167$.

Cohen J (1969). Statistical power analysis for the behavioural sciences. New York: Academic Press.

Crick NR and Dodge KA (1994). A review and reformulation of social information-processing mechanisms in children's social adjustment. Psychological Bulletin, 115, 74-101.

Dillon KH, Allan NP, Cougle JR and Fincham FD (2016). Measuring hostile interpretation bias: The WSAP-hostility scale. Assessment, 23, 707-719.

Farrar K and Krcmar M (2006). Measuring state and trait aggression: A short, cautionary tale. Media Psychology, 8, 127-138.

Faul F, Erdfelder E, Lang A-G and Buchner A (2007). G*Power 3: A flexible statistical power analysis program for the social, behavioral, and biomedical sciences. Behavior Research Methods, 39, 175-191.

Ferrari GR, Möbius M, van Opdorp A, Becker ES and Rinck M (2016). Can't look away: An eye-tracking based attentional disengagement training for depression. Cognitive Therapy and Research, 40, 672-686.

Foulsham T, Gray A, Nasiopoulos E and Kingstone A (2013). Leftward biases in picture scanning and line bisection: A gaze-contingent window study. Vision Research, 78, 14-25.

Gerdes AB, Alpers GW and Pauli P (2008). When spiders appear suddenly: Spider-phobic patients are distracted by task-irrelevant spiders. Behaviour Research and Therapy, 46, 174-187.

Hallion LS and Ruscio AM (2011). A meta-analysis of the effect of cognitive bias modification on anxiety and depression. Psychological Bulletin, 137, 940-958.

Hawkins KA and Cougle JR (2013). Effects of interpretation training on hostile attribution bias and reactivity to interpersonal insult. Behavior Therapy, 44, 479-488.

Heeren A, Mogoașe C, Philippot P and McNally RJ (2015). Attention bias modification for social anxiety: A systematic review and meta-analysis. Clinical Psychology Review, 40, 76-90.

Hiemstra W, de Castro BO and Thomaes S (2018). Reducing aggressive children's hostile attributions: A Cognitive Bias Modification Procedure. Cognitive Therapy and Research, 43, 387-398.

Hirsch CR and Clark DM (2004). Information-processing bias in social phobia. Clinical Psychology Review, 24, 799-825.

Hirsch CR, Clark DM and Mathews A (2006). Imagery and interpretations in social phobia: Support for the combined cognitive biases hypothesis. Behavior Therapy, 37, 223-236.

Holmes EA, Lang TJ and Shah DM (2009). Developing interpretation bias modification as a "cognitive vaccine" for depressed mood: Imagining positive events makes you feel better than thinking about them verbally. Journal of Abnormal Psychology, 118, 76-88.

Horsley TA, de Castro BO and Van der Schoot M (2010). In the eye of the beholder: Eye-tracking assessment of social information processing in aggressive behavior. Journal of Abnormal Child Psychology, 38, 587-599.

Huijding J, Mayer B, Koster EH and Muris P (2011). To look or not to look: An eye movement study of hypervigilance during change detection in high and low spider fearful students. Emotion, 11, 666-674.

Lazarov A, Pine DS and Bar-Haim Y (2017). Gaze-contingent music reward therapy for social anxiety disorder: A randomized controlled trial. American Journal of Psychiatry, 174, 649-656.

Lisk SC, Pile V, Haller SP, Kumari V and Lau JY (2018). Multisession cognitive bias modification targeting multiple biases in adolescents with elevated social anxiety. Cognitive Therapy and Research, 42, 581-597.

MacLeod C and Clarke PJ (2015). The attentional bias modification approach to anxiety intervention. Clinical Psychological Science, 3, 58-78.

MacLeod C, Rutherford E, Campbell L, Ebsworthy G and Holker L (2002). Selective attention and emotional vulnerability: Assessing the causal basis of their association through the experimental manipulation of attentional bias. Journal of Abnormal Psychology, 111, 107-123.

Mathews A and Mackintosh B (2000). Induced emotional interpretation bias and anxiety. Journal of Abnormal Psychology, 109, 602-615.

Naim R, Kivity Y, Bar-Haim Y and Huppert JD (2018). Attention and interpretation bias modification treatment for social anxiety disorder: A randomized clinical trial of efficacy and synergy. Journal of Behavior Therapy and Experimental Psychiatry, 59, 19-30.

Novaco RW (1994). Anger as a risk factor for violence among the mentally disordered. In J Monahan and $\mathrm{H}$ Steadman (Eds.), Violence and mental disorder: Developments in risk assessment (pp. 21-59). Chicago: University of Chicago Press.

Panagiotidis D, Clemens B, Habel U, Schneider F, Schneider I, Wagels L and Votinov M (2017). Exogenous testosterone in a non-social provocation paradigm potentiates anger but not behavioral aggression. European Neuropsychopharmacology, 27, 1172-1184. 
Penton-Voak IS, Thomas J, Gage SH, McMurran M, McDonald S and Munafò MR (2013). Increasing recognition of happiness in ambiguous facial expressions reduces anger and aggressive behavior. Psychological Science, 24, 688-697.

Price RB, Greven IM, Siegle GJ, Koster EH and De Raedt R (2016). A novel attention training paradigm based on operant conditioning of eye gaze: Preliminary findings. Emotion, 16, 110-116.

Raine A, Dodge K, Loeber R, Gatzke-Kopp L, Lynam D, Reynolds C, ... Liu J (2006). The reactive-proactive aggression questionnaire: Differential correlates of reactive and proactive aggression in adolescent boys. Aggressive Behavior, 32, 159-171.

Sanchez-Lopez A, De Raedt R, van Put J and Koster EH (2019). A novel process-based approach to improve resilience: Effects of computerized mouse-based (gaze) contingent attention training (MCAT) on reappraisal and rumination. Behaviour Research and Therapy, 118, 110-120.

Sanchez A, Everaert J and Koster EH (2016). Attention training through gaze-contingent feedback: Effects on reappraisal and negative emotions. Emotion, 16, 1074-1085.

Schönenberg M and Jusyte A (2014). Investigation of the hostile attribution bias toward ambiguous facial cues in antisocial violent offenders. European Archives of Psychiatry and Clinical Neuroscience, 264, 61-69.

Troop-Gordon W, Gordon RD, Vogel-Ciernia L, Ewing Lee E and Visconti KJ (2018). Visual attention to dynamic scenes of ambiguous provocation and children's aggressive behavior. Journal of Clinical Child \& Adolescent Psychology, 47, 925-940.

Vassilopoulos SP, Brouzos A and Andreou E (2015). A multi-session attribution modification program for children with aggressive behavior: Changes in attributions, anger score estimates, and self-reported aggression. Behavioral and Cognitive Psychotherapy, 43, 538-548.

Watson D, Clark LA and Tellegen A (1988). Development and validation of brief measures of positive and negative affect: The PANAS scales. Journal of Personality and Social Psychology, 54, 1063-1070.

White LK, Suway JG, Pine DS, Bar-Haim Y and Fox NA (2011). Cascading effects: The influence of attention bias to threat on the interpretation of ambiguous information. Behaviour Research and Therapy, 49, 244-251.

Wilkowski BM and Robinson MD (2008). The cognitive basis of trait anger and reactive aggression: An integrative analysis. Personality and Social Psychology Review, 12, 3-21.

Wilkowski BM, Robinson MD, Gordon RD and Troop-Gordon W (2007). Tracking the evil eye: Trait anger and selective attention within ambiguously hostile scenes. Journal of Research in Personality, 41, 650-666.

World Medical Association (2001). Declaration of Helsinki World Medical Association Declaration of Helsinki. Bulletin of the World Health Organization, 79, 373-374.

Cite this article: AlMoghrabi N, Franken IHA, Mayer B, Huijding J (2022). A Single-Session Combined Cognitive Bias Modification Training Targeting Attention and Interpretation Biases in Aggression. Behaviour Change 39, 1-20. https:// doi.org/10.1017/bec.2021.11 